\title{
REAÇÕES DE POLIMERIZAÇÃO DE MMA EM MINIEMULSÃO COM AQUECIMENTO MICRO-ONDAS. EFEITO NA DISTRIBUIÇÃO DE MASSA MOLAR
}

\author{
E. T. S. ALVES ${ }^{1}$, I. M. S. NICÁCIO ${ }^{2}$, P. H. H. ARAUJO ${ }^{1}$, A. F. SANTOS ${ }^{2}$, C. SAYER ${ }^{1}$ \\ ${ }^{1}$ Universidade Federal de Santa Catarina, Departamento de Engenharia Química e Engenharia de \\ Alimentos UFSC - SC \\ ${ }^{2}$ Universidade Tiradentes, Instituto de Tecnologia e Pesquisa UNIT - SE \\ E-mail para contato: ediely.tsa@gmail.com
}

\begin{abstract}
RESUMO - Neste trabalho foi avaliado o efeito do aquecimento micro-ondas (MO) sobre a massa molar de PMMA preparado por polimerização em miniemulsão com iniciadores hidrossolúvel (KPS) e organossolúvel (AIBN), em comparação ao aquecimento convencional. Os resultados mostram que nas reações com AIBN a massa molar do PMMA foi menor no reator MO. Nas reações com KPS, por outro lado, o PMMA apresentou massas molares parecidas entre o aquecimento convencional e MO, apesar da maior velocidade de reação no reator MO.
\end{abstract}

\section{INTRODUÇÃO}

O processo de polimerização em miniemulsão vem sendo amplamente difundido na síntese de nanopartículas poliméricas. A polimerização em miniemulsão se destaca por apresentar vantagens como o uso não excessivo de tensoativo, estabilidade coloidal suficiente e incorporação de compostos hidrofóbicos (ROMIO et al., 2009; TIARKS et al., 2001). Tais vantagens se devem, principalmente, ao mecanismo de nucleação das gotas e a polimerização destas. Na polimerização em miniemulsão o monômero encontra-se pré-disperso em gotas submicrométricas de 50 - $500 \mathrm{~nm}$, que são estabilizadas pela combinação entre um tensoativo e co-estabilizador. Nesta técnica é necessário um mecanismo de cisalhamento (homogeneização) para garantir o tamanho submicrometrico das gotas. O uso do coestabilizador retarda a degradação difusional que ocorre devido a alta energia de cisalhamento aplicada ao sistema (TIARKS et al., 2001. O outro aspecto relevante na obtenção de uma miniemulsão estável é minimizar a coalescência por coalisão das gotas monoméricas, para isto, a escolha adequada do tipo e quantidade de tensoativo é fundamental (TIARKS et al., 2001).

Uma alternativa promissora na condução de reações de polimerização é a utilização da tecnologia micro-ondas (MO) em substituição ao aquecimento convencional (COSTA et al., 2011; HOLTZE et al., 2006). De forma geral, o aquecimento por micro-ondas ocorre devido a polarização dipolar das moléculas, que são permanentemente polarizadas por ligações químicas e realinhadas na presença de campo elétrico de alta frequência (LIDSTROM et al., 2001). Como resultado, a distribuição de temperatura é mais uniforme e torna os fenômenos térmicos clássicos, condução e 
convecção, como secundários. Entretanto, ainda são escassos os estudos envolvendo micro-ondas aplicadas ao processo de polimerização em miniemulsão, motivando assim, o desenvolvimento deste trabalho, que tem como foco principal a avaliação do efeito das micro-ondas sobre a distribuição de massa molar de nanopartículas biocompatíveis obtidas pela polimerização em miniemulsão do MMA utilizando lecitina de soja, Crodamol GTCC e KPS ou AIBN como tensoativo, co-estabilizador e iniciador, respectivamente.

\section{MATERIAIS E MÉTODOS}

\subsection{Materiais}

O monômero utilizado foi o metacrilato de metila (MMA) da Merk. O crodamol GTCC da Alpha Química e a lecitina da Alfa Aesar, ambos biodegradáveis e biocompativeis, foram aplicados como co-estabilizador e tensoativo, respectivamente. Os iniciadores empregados foram o aniônico persulfato de potássio (KPS) da Sigma-Aldrich e o organossolúvel - 2,2-azo-bis-isobutironitrila (AIBN, 98\% puro) da DuPont. A água destilada foi utilizada como meio contínuo. Todos reagentes foram utilizados como fornecidos, com exceção do AIBN que foi recristalizado. Para cessar a reação instantaneamente no momento da amostragem foi utilizada uma solução de hidroquinona (1\% p/p), previamente preparada a partir de hidroquinona sólida da marca Dinâmica.

\subsection{Reator Micro-ondas}

As reações conduzidas sob aquecimento MO foram realizadas no reator micro-ondas Synthos 3000 da Anton Paar. A energia micro-ondas operou a uma frequência de 2,45 GHz e potência máxima de $1400 \mathrm{~W}$. A unidade é composta por um rotor contendo oito frascos de quartzo $(80 \mathrm{~mL})$ e agitador magnético. A temperatura foi monitorada por um sensor a gás inserido na fase líquida de um frasco de referência e por um sensor de infravermelho localizado na parte inferior dos frascos. A Figura 1 apresenta o reator micro-ondas utilizado e o rotor giratório do reator com os frascos de quartzo.

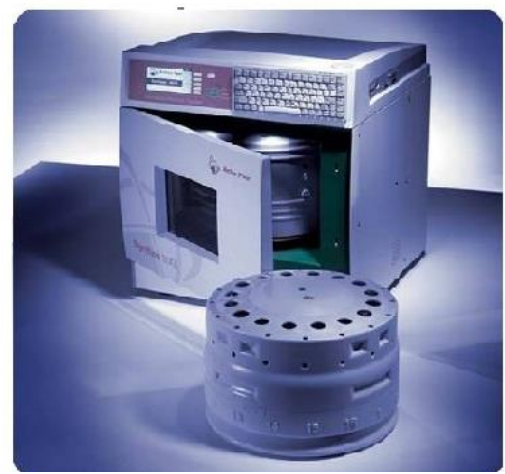

(a)

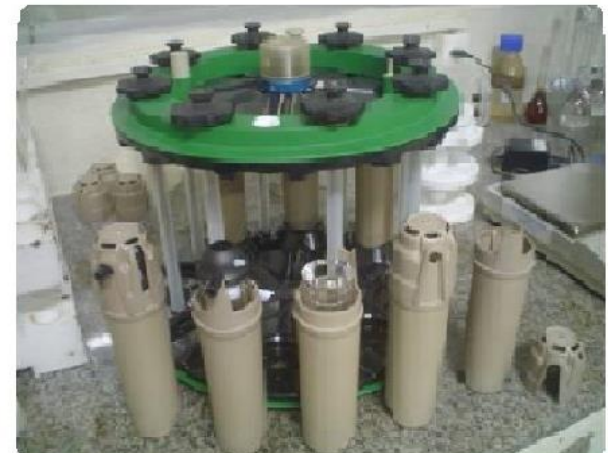

(b)

Figura 1 - Reator Micro-ondas. (a) cavidade (b) detalhe do rotor para inserção de 8 frascos. 


\subsection{Polimerização em Miniemulsão}

Primeiramente misturou-se por agitação magnética a lecitina, Crodamol GTCC e AIBN (se necessário). O MMA foi adicionado à fase orgânica e a agitação prosseguiu por 5 min com banho de gelo, a fim de evitar a evaporação do monômero. Em seguida, misturaram-se as fases orgânica e aquosa por agitação magnética por mais $20 \mathrm{~min}$. Na etapa seguinte ocorreu a homogeneização da emulsão por ultrassom acoplado de uma ponteira (Fisher Scientific, Sonic Dismembrator Model 500, $400 \mathrm{~W}$ ) por 4 min na amplitude de $60 \%$. Ao fim da etapa de dispersão, a miniemulsão foi transferida para o reator. Nas reações com KPS, este foi adicionado a miniemulsão logo após a dispersão. Todas as reações foram realizadas à temperatura constante, $70^{\circ} \mathrm{C}$. Periodicamente, amostras de $5 \mathrm{~mL}$ foram retiradas para análises da conversão e tamanho de partícula.

\subsection{Caracterização}

Conversão: A conversão foi determinada por análise gravimétrica.

Tamanho de partícula: O diâmetro médio (média z) das partículas foi determinado por Dispersão Dinâmica de Luz utilizando o equipamento Zetasizer Nano Series da Malvern Instruments. Para tanto, a amostra foi diluída em água saturada contendo $1 \%$ de metacrilato de metila.

Massa molar: A massa molar dos polímeros foi determinada por cromatografia de permeação em gel (GPC) em equipamento da marca Shimadzu, (LC-20A). As amostras foram preparadas a partir da diluição de $20 \mathrm{mg}$ de polímero seco em $4 \mathrm{~mL}$ de tetrahidrofurano (THF, Vetec Química Fina LTDA). Em seguida, as soluções foram filtradas em filtro de Nylon $(\mathrm{d}=0,45 \mu \mathrm{m})$. As soluções filtradas foram injetadas à temperatura ambiente e analisadas a $35^{\circ} \mathrm{C}$. A curva de calibração foi baseada em padrões de poliestireno com massas molares variando entre $580 \mathrm{~g} / \mathrm{gmol}$ e $3,8 \times 10^{6} \mathrm{~g} / \mathrm{gmol}$.

\section{RESULTADOS}

As condições experimentais das reações de polimerização em miniemulsão de MMA sob aquecimento convencional ou MO são apresentadas na Tabela 1. Os ensaios foram realizados sob distintos tipos e concentrações de iniciadores.

Tabela1 - Formulações das reações de polimerização à $70^{\circ} \mathrm{C}$.

\begin{tabular}{lcccc}
\hline \multirow{2}{*}{ Reagentes } & \multicolumn{4}{c}{ Reações $(\%$ m/m em relação a massa total $)$} \\
\cline { 2 - 5 } & K1 & K2 & A1 & A2 \\
\hline Água & 79,98 & 79,95 & 80,00 & 80,00 \\
Lecitina & 0,30 & 0,29 & 0,29 & 0,29 \\
Crodamol GTCC & 9,84 & 9,83 & 9,80 & 9,73 \\
MMA & 9,84 & 9,83 & 9,80 & 9,73 \\
KPS & 0,02 & 0,05 & - & - \\
AIBN & - & - & 0,1 & 0,24 \\
\hline
\end{tabular}




\subsection{Comparação entre o Aquecimento Convencional e MO com KPS como Iniciador}

As Figuras 2 (a) e (b) apresentam a evolução da conversão durante reações de polimerização em miniemulsão de MMA com aquecimento convencional e MO para as formulações K1 $(0,02 \%$ de KPS m.m. $\left.\mathrm{m}_{\mathrm{T}}\right)$ e K2 (0,05\% de KPS m. $\mathrm{m}_{\mathrm{T}}$.), respectivamente. Observa-se um aumento na velocidade de reação para as reações assistidas por MO quando comparadas ao aquecimento convencional, consequentemente, houve redução de $50 \%$ do tempo de reação. Além disso, o efeito das micro-ondas na cinética da reação foi mais expressivo na reação com menor concentração de iniciador (K1_MO). De acordo com Costa e colaboradores (2009), este efeito na cinética da reação se deve ao aumento da velocidade de decomposição térmica do KPS, visto que, a constante cinética de decomposição do KPS por MO $\left(\mathrm{k}_{\mathrm{dMO}}\right)$ é 3,64 maior que $\mathrm{k}_{\mathrm{d} C O N V}$.

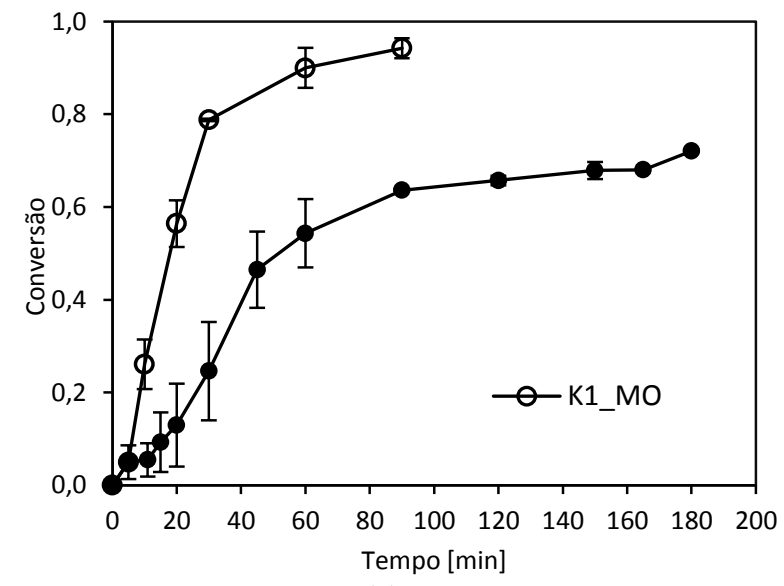

(a)

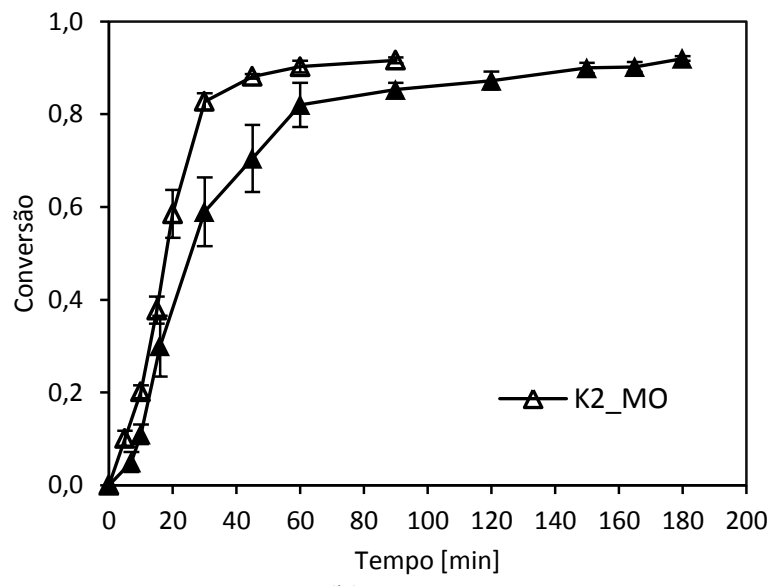

(b)

Figura 2 - Evolução da conversão. (a) reações K1 e (b) reações K2.

$\mathrm{Na}$ reação K1 com MO foram obtidas partículas menores do que aquelas em reator convencional como pode ser visto nas Figuras 3 (a) e (b). Esta diminuição no diâmetro das partículas pode ser atribuída à elevada velocidade de decomposição térmica do iniciador promovida pela radiação $\mathrm{MO}$, que por sua vez, contribui para a nucleação de um grande número de partículas e, como consequência, para obtenção de tamanhos de partícula menores. Entretanto, na formulação K2 os diâmetros de partículas foram semelhantes entre os dois modos de aquecimento. Tal resultado indica que o maior número partículas nucleadas não é o único responsável pelo aumento da velocidade de polimerização nas reações com MO. Outro fato observado foi a variação do tamanho das partículas no início da reação. O que pode indicar ocorrência da nucleação homogênea, provavelmente devido a solubilidade do KPS e MMA na fase aquosa. 


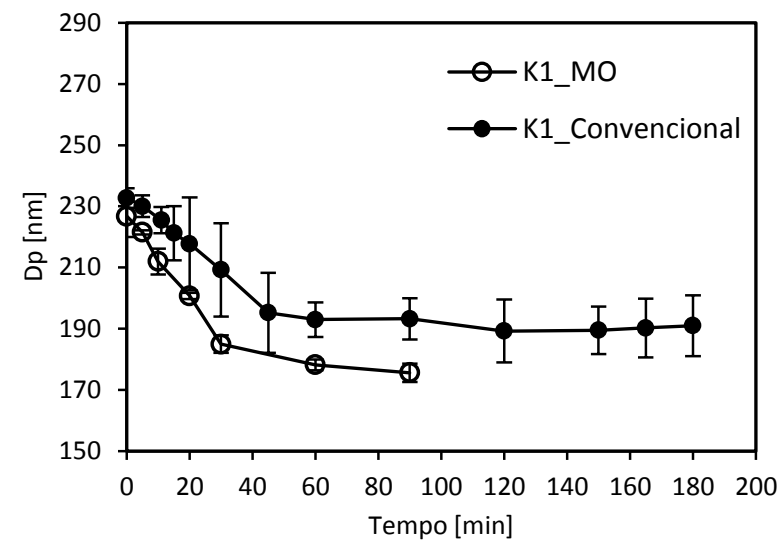

(a)

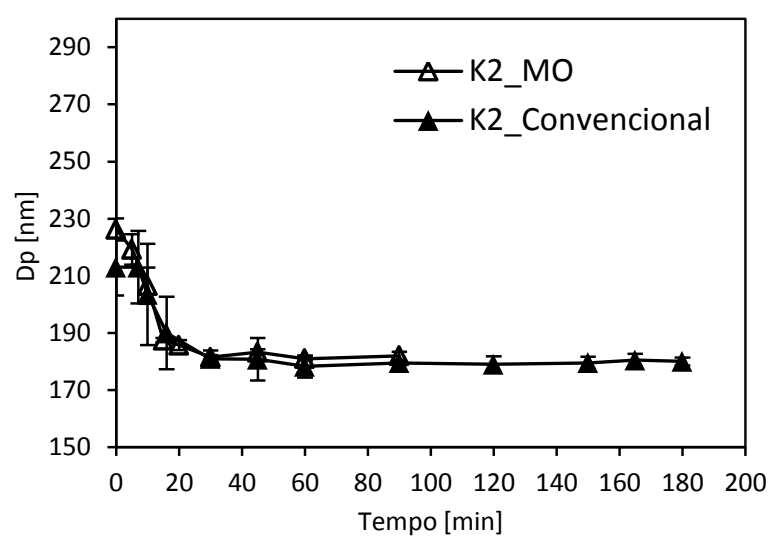

(b)

Figura 3 - Evolução do diâmetro médio de partícula. (a) reações K1 e (b) reações K2.

Apesar das reações conduzidas por MO apresentarem maior velocidade de reação, não foram observadas diferenças significativas quanto a distribuição da massa molar e seus valores médios, conforme pode ser visto na Tabela 2 e Figura 4. Este resultado surpreende, visto que, os tamanhos médios das partículas foram semelhantes e a maior velocidade de geração dos radicais na reação com MO, o que resulta numa maior frequência de entrada de radicais nas partículas e, por consequência, o comportamento esperado seria que resultasse em massas molares menores. A ausência deste comportamento pode ser um indicativo de que o aquecimento micro-ondas afeta (negativamente) as reações de terminação.

Tabela 2 - Comparação entre a conversão limite (X), diâmetro médio da gotas (Dg) e partícula (Dp), massa molar média em número (Mn) e em massa (Mw) e o índice de polidispersão (IP).

\begin{tabular}{|c|c|c|c|}
\hline Látex & Reator & Conversão(\%) & $\mathrm{D}_{\mathrm{g}}(\mathrm{nn}$ \\
\hline \multirow[t]{2}{*}{ K1 } & \multirow{2}{*}{$\begin{array}{c}\text { Convencional } \\
\text { MO }\end{array}$} & $72 \pm 1$ & $233 \pm 3$ \\
\hline & & $90 \pm 2$ & $227 \pm 7$ \\
\hline \multirow{2}{*}{ K2 } & \multirow{2}{*}{$\begin{array}{c}\text { Convencional } \\
\text { MO }\end{array}$} & $93 \pm 3$ & $213 \pm 1$ \\
\hline & & $92 \pm 1$ & $226 \pm 4$ \\
\hline \multirow{4}{*}{$\begin{array}{l}\sum_{00}^{3} \\
\frac{0}{3} \\
\frac{3}{0}\end{array}$} & $\mathrm{C}_{\mathrm{K} 1}$ & NVENCIONAL & \\
\hline & ${ }_{-\ldots} \mathrm{K}_{-}$ & & \\
\hline & 0,2 & & \\
\hline & 3 & ${ }^{5} \log M w^{6}$ & 8 \\
\hline
\end{tabular}

Figura 4 - Distribuição da massa molar do látex final (a) reações K1 e (b) reações K2. 


\subsection{Comparação entre o Aquecimento Convencional e MO com AIBN como Iniciador}

Na evolução da cinética de reação, observou-se maior velocidade de reação para as formulações estudadas (A1, A2), conforme pode ser visto nas Figuras 5 (a) e (b) Na reação A2 (0,24\% de AIBN m. $\mathrm{m}_{\mathrm{T}}$ ), o efeito das MO sobre a velocidade de reação diminui ao longo da reação, atingindo igual conversão limite no mesmo tempo que a reação convencional. Ergan e Bayramoglu (2013) constataram em estudo sobre a decomposição do AIBN com aquecimento MO, o aumento do fator pré-exponencial e da energia de ativação em comparação ao aquecimento convencional. Entretanto, os autores não alcançaram uma relação clara entre a energia aplicada no reator MO e o valor da constante de decomposição do AIBN.

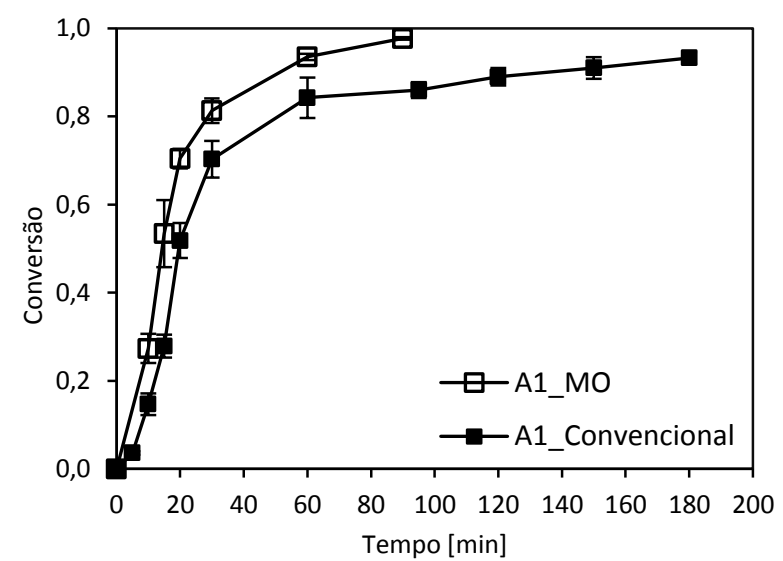

(a)

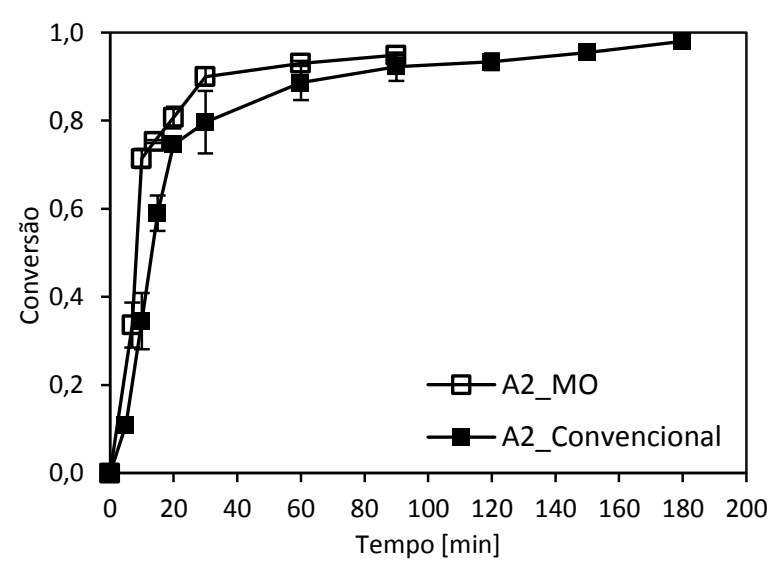

(b)

Figura 5 - Evolução da conversão. (a) reações A1 e (b) reações A2.

Na reação com aquecimento MO, A1 e A2, apesar da maior velocidade de reação, os diâmetros médios das partículas foram semelhantes aos valores obtidos na reação convencional, conforme mostra a Figura 6. Portanto, o aumento da velocidade de reação com micro-ondas não pode ser atribuído apenas às diferenças dos tamanhos das partículas.

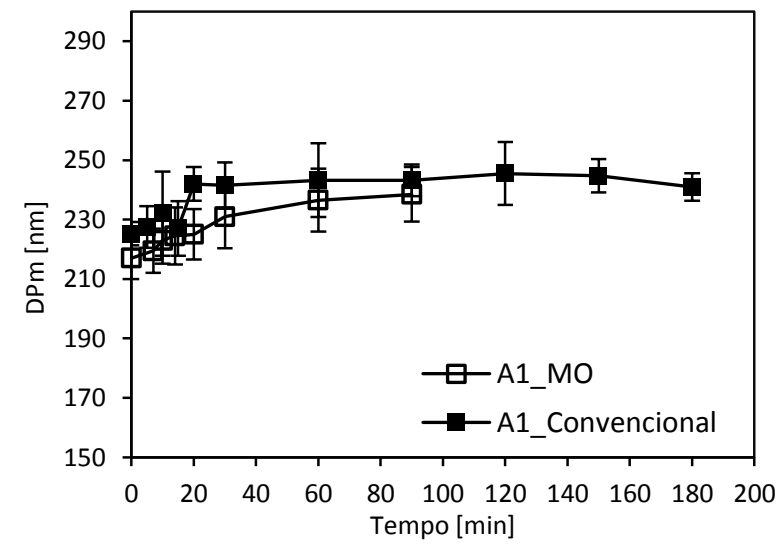

(a)

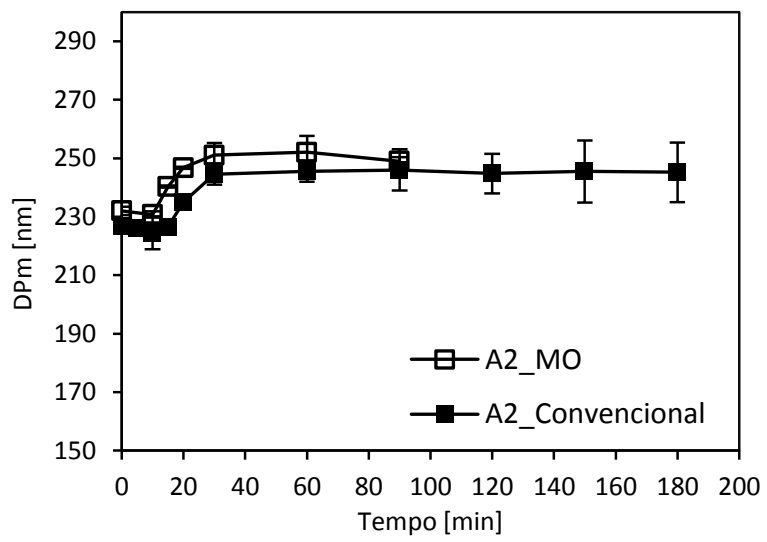

(b)

Figura 6 - Evolução do diâmetro médio de partícula. (a) reações A1 e (b) reações A2. 
Além das diferenças cinéticas observadas nas as reações com AIBN, observou-se também, a variação da massa molar para as duas formulações (A1 e A2) entre os modos de aquecimento, como mostram a Figura 7 e Tabela 3. A curva de distribuição da massa molar apresentou deslocamento para valores mais baixos, assim como, os valores médios de massa molar numérica e ponderal para os látices obtidos por aquecimento micro-ondas. Resultado esperado devido a maior velocidade de geração dos radicais.

Tabela 3 - Comparação entre a conversão limite $(\mathrm{X})$, diâmetro médio da gotas (Dg) e partícula (Dp), massa molar média em número $(\mathrm{Mn})$ e em massa (Mw) e o índice de polidispersão (IP).

\begin{tabular}{lccccccc}
\hline Látex & Reator & $\begin{array}{c}\text { Conversão } \\
(\%)\end{array}$ & $\begin{array}{c}\mathrm{Dg} \\
(\mathrm{nm})\end{array}$ & $\begin{array}{c}\mathrm{Dp} \\
(\mathrm{nm})\end{array}$ & $\begin{array}{c}\text { Mnx105 } \\
(\mathrm{g} / \mathrm{mol})\end{array}$ & $\begin{array}{c}\text { Mwx105 } \\
(\mathrm{g} / \mathrm{mol})\end{array}$ & $\mathrm{IP}$ \\
\cline { 2 - 5 } $\mathrm{A} 1$ & Convencional & $92 \pm 3$ & $227 \pm 2$ & $245 \pm 10$ & 5,64 & 1,27 & 2,25 \\
& MO & $98 \pm 2$ & $232 \pm 2$ & $250 \pm 2$ & 3,19 & 7,75 & 2,43 \\
\multirow{2}{*}{$\mathrm{A} 2$} & Convencional & $98 \pm 2$ & $225 \pm 4$ & $236 \pm 5$ & 2,11 & 8,33 & 3,94 \\
& MO & $95 \pm 2$ & $215 \pm 7$ & $230 \pm 10$ & 1,63 & 4,97 & 3,05 \\
\hline
\end{tabular}
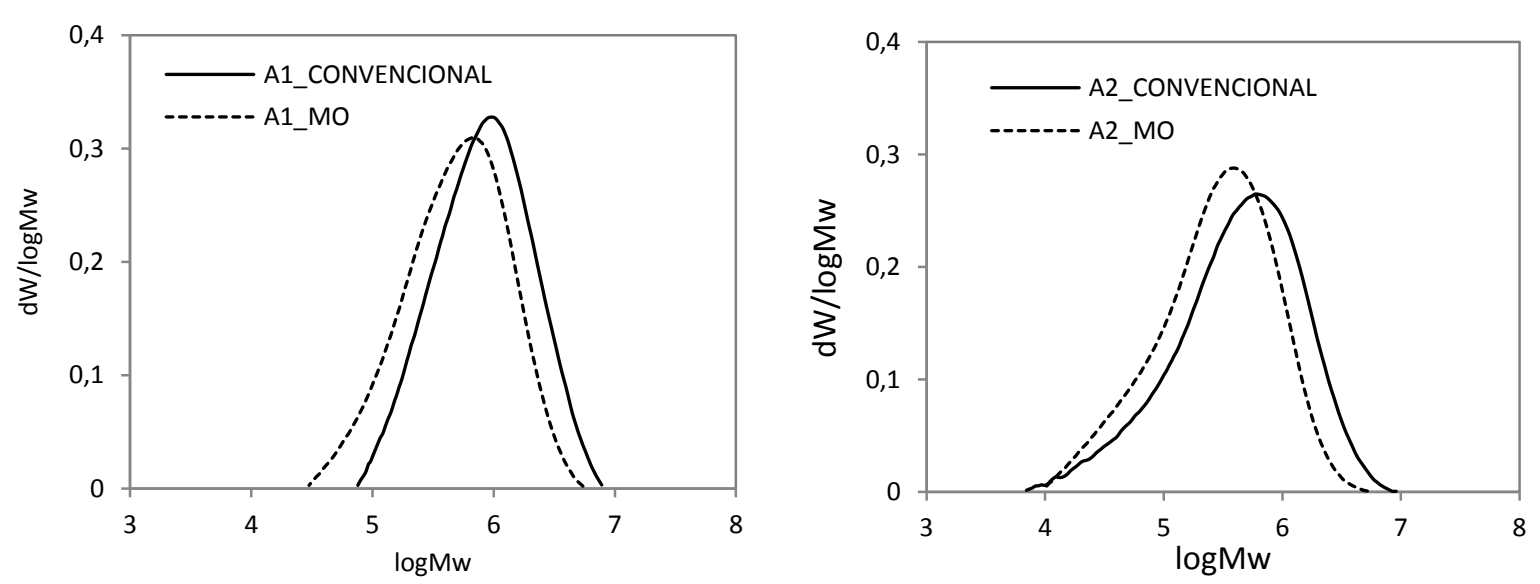

Figura 7 - Distribuiçấo da massa molar do látex final (a) reações A1 (b) (b) reações A2.

\section{CONCLUSÕES}

De modo geral, a utilização da radiação MO é uma alternativa promissora para melhorar o processo de polimerização em miniemulsão de MMA, possibilitando a formação de nanocápsulas sem interferir nas características de tamanho das partículas e, com grande redução de energia e tempo em relação ao aquecimento convencional. No entanto, as massas molares obtidas dos látices com AIBN como iniciador apresentaram alteração na sua distribuição para uma massa molar menor. Já com o KPS, as massas molares mantiveram seus valores médios semelhantes ao aquecimento convencional. Sugere-se que os efeitos micro-ondas predominantes nas reações estudadas podem estar relacionados aos efeitos térmicos, decorrentes da presença de pontos superaquecidos dentro da amostra e da seletividade das substâncias presentes no meio reacional. 


\section{REFERÊNCIAS}

C. COSTA, V.H.S. SANTOS, C. SAYER, P.H.H. ARAÚJO, A.F. SANTOS, M. FORTUNY. Eup. Polymr J. v. 45, p. 2011-2016, 2009.

COSTA, C., SANTOS, V. H. S., SAYER, C. ARAUJO, P. H. H., SANTOS, A. F., FORTUNY, M. Microwave effects due to anionic or cationic initiators in emulsion polymerization reactions. Macromol. Symp., v. 302, p. 161-168, 2011.

ERGAN, B.T., BAYRAMOGLU, M. The effects of microwave power and dielectric properties on the microwave-assisted decomposition kinetics of AIBN in n-butanol. Ind Eng Chem, v.19, p.299-304, 2013.

LIDSTROM, P., TIERNEY, J., WATHEY, B., WESTMAN, J. Microwave assisted organic synthesis - A review. Tetrahedron, v. 57, p. 9225-9283, 2001.

ROMIO, A. P.; SAYER,C.; ARAÚJO, P. H. H.; AL-HAYDARI, M.; WU, L.; Da ROCHA, S. R. P. Nanocapsules by Miniemulsion Polymerization with Biodegradable Surfactant and Hydrophobe. Macromol. Chem. Phys,v. 210, p. 747-75, 2009.

TIARKS, F.; LANDFESTER, K.; ANTONIETTI, M. preparation of polymeric nanocapsules by miniemulsion polymerization. Langmuir, v. 17, p. 908-918, 2001. 